\title{
The Rational Toomer invariant and Certain Elliptic Spaces
}

\author{
Gregory Lupton
}

\begin{abstract}
We give an explicit formula for the rational category of an elliptic space whose minimal model has a homogeneous-length differential. We also show that for such a space, there are no gaps in the sequence of integers realized as the rational Toomer invariant of some cohomology class. With an additional hypothesis, we show a result from which we deduce the relation $\operatorname{dim}\left(H^{*}(X ; \mathbb{Q})\right) \geq 2 \operatorname{cat}_{0}(X)$.
\end{abstract}

\section{Introduction}

Since the fundamental paper of Félix and Halperin [FH82], the so-called $r a-$ tional Toomer invariant of a space has played a central role in the development of results concerning rational category. The rational Toomer invariant of a space $X$ is a numerical rational homotopy invariant, denoted by $\mathrm{e}_{0}(X)$, that provides a lower bound for the rational category of a space. We recall its definition below. In general, its value is (strictly) between the rational cup length and the rational category of the space. One can also consider the rational Toomer invariant of an individual cohomology class. This is a finer invariant, whose supremum, taken over all cohomology classes of a space, retrieves the rational Toomer invariant of the space. The rational Toomer invariant of a cohomology class can also be identified with the strict, or essential, category weight of the cohomology class, in the sense of Rudyak and Strom (cf. [Rud99, Str97]). Since we are only concerned with the rational case, we will henceforth refer to the rational Toomer invariant simply as the Toomer invariant.

In this paper, we study the (rational) Toomer invariant for rationally elliptic spaces. Recall that a simply connected space $X$ is called rationally elliptic if both $H^{*}(X ; \mathbb{Q})$ and $\pi_{*}(X) \otimes \mathbb{Q}$ are finite-dimensional vector spaces. For these spaces, the Toomer invariant becomes all the more interesting, since it is known that cat ${ }_{0}(X)=$ $\mathrm{e}_{0}(X)$ for any elliptic space $X$, where $\operatorname{cat}_{0}(X)$ denotes the rational category of the space $X$. Indeed, it is proved more generally in [FHL98] that cat ${ }_{0}(X)=\mathrm{e}_{0}(X)$ for any space $X$ that satisfies rational Poincaré duality.

2000 Mathematics Subject Classification. Primary 55P62, 55M30; Secondary 55T10.

Key words and phrases. Rational category, elliptic space, Toomer invariant, $\mathrm{e}_{0}$-invariant, Moore length, minimal model, Wang sequence, Gysin sequence.

(C)2002 American Mathematical Society 
We now outline our results and indicate the organization of the paper. The main result is Theorem 2.2. We assume that the minimal model of an elliptic space has a homogeneous-length differential. Then part (A) of Theorem 2.2 gives a formula for the Toomer invariant, and hence the rational category, of the elliptic space. The approach used to prove this part of the result leads to finer information about the Toomer invariant of individual cohomology classes. Paraphrasing the precise statement, part (B) of Theorem 2.2 says that for an elliptic space whose minimal model has a homogeneous-length differential, there are no gaps in the sequence of integers realized as the Toomer invariant of some cohomology class. Part (C) of the result gives information about the location of cohomology classes that have a given Toomer invariant. Our second result is Theorem 2.5. Here we show that under a fairly mild additional hypothesis, there are at least two linearly independent cohomology classes with Toomer invariant any integer strictly between zero and the Toomer invariant of the space. The inequality $\operatorname{dim}\left(H^{*}(X ; \mathbb{Q})\right) \geq 2$ cat $_{0}(X)$ results. The paper ends with a brief section of examples and comments.

A Little Archaeology and Acknowledgements: The formula of part (A) of Theorem 2.2 can be adapted into a lower bound for the rational category of any elliptic space - cf. Remark 2.4. Indeed, an earlier version of this paper included this as a separate result. During the refereeing process, however, it was brought to my attention that this lower bound appears as Corollary 3 in [GJ01]. Further, the methods used there to obtain the lower bound, amongst a number of other interesting related results, are comparable to the methods of this paper. A forthcoming paper [CJ01] also contains a similar, but more general result. The referee further pointed out to me that part (A) of Theorem 2.2 appears as a special case of Theorem 1 (Theorem 7) of [LM01]. Their method of proof in that paper, however, is substantially different from the one given here and does not yield the finer information given by parts (B) and (C) of Theorem 2.2. I thank for the referee for bringing these articles to my attention.

We finish this introductory section with a brief review of some ideas from rational homotopy theory. All results of this paper are proved using standard tools of the subject. We refer to [FHT01] for a general introduction to these techniques. We recall some of the notation here. By a minimal algebra we mean a free graded commutative algebra $\Lambda V$, for some finite-type graded vector space $V$, together with a differential $d$ of degree +1 that is decomposable, i.e., satisfies $d: V \rightarrow \Lambda^{\geq 2} V$. We assume that the minimal algebra is simply connected, i.e., that the vector space $V$ has no generators in degrees lower than 2. This assumption is necessary in order to translate our algebraic results into topological ones, although it is not strictly necessary for the algebraic results themselves. A minimal algebra $\Lambda V$ is called elliptic if both $V$ and the cohomology algebra $H^{*}(\Lambda V)$ are finite-dimensional vector spaces. If $\left\{v_{1}, \ldots, v_{n}\right\}$ is a graded basis for $V$, then we write $\Lambda V$ as $\Lambda\left(v_{1}, \ldots, v_{n}\right)$. A basis can always be chosen so that $d v_{1}=0$ and for $i \geq 2, d v_{i} \in \Lambda\left(v_{1}, \ldots, v_{i-1}\right)$.

Every simply connected space with rational cohomology of finite-type has a corresponding minimal model, which is a minimal algebra that encodes the rational homotopy of the space. Although our results are stated and proved in purely algebraic terms, they do admit topological interpretations via this correspondence. In particular, a simply connected space is rationally elliptic if its minimal model is an elliptic minimal algebra. Because of the correspondence between spaces and 
their minimal models, this characterization of a rationally elliptic space coincides with that given earlier.

We recall the definition, in minimal algebra terms, of the Toomer invariant for a cohomology class and for a space. Suppose that $\Lambda V$ is a minimal algebra. For $n \geq 1$, let $p_{n}$ denote projection onto the quotient differential graded (DG) algebra obtained by factoring out the DG ideal generated by monomials of length at least $n+1$, thus

$$
p_{n}: \Lambda V \rightarrow \frac{\Lambda V}{\Lambda \geq n+1 V}
$$

Define $\mathrm{e}_{0}(\Lambda V)$ to be the smallest $n$ such that $p_{n}$ induces an injection on cohomology, or set $\mathrm{e}_{0}(\Lambda V)=\infty$ if there is no such smallest $n$ (cf. [FHT01, p.381]). This can be extended to apply to individual cohomology classes as follows: Suppose $x \in H^{*}(\Lambda V)$ is some fixed, non-zero cohomology class. For obvious degree reasons, $p_{n}^{*}(x) \neq 0$ for large enough $n$. We define $\mathrm{e}_{0}(x)$ to be the smallest $n$ for which $p_{n}^{*}(x) \neq 0$. If the set of integers $\left\{\mathrm{e}_{0}(x) \mid x \neq 0 \in H^{*}(\Lambda V)\right\}$ has a maximum, then we see that $\mathrm{e}_{0}(\Lambda V)$ is this maximum. Otherwise we have $\mathrm{e}_{0}(\Lambda V)=\infty$.

Where convenient, we will use a standard observation about $\mathrm{e}_{0}(\Lambda V)$ for a minimal algebra $\Lambda V$ whose cohomology satisfies Poincaré duality. Namely, that if $\mu$ denotes a fundamental class of $H^{*}(\Lambda V)$, then $\mathrm{e}_{0}(\Lambda V)=\mathrm{e}_{0}(\mu)$ [FH82, Lem.10.1]. It is well-known that an elliptic minimal algebra satisfies Poincaré duality [Hal77, Th.3]. By the formal dimension of an elliptic minimal algebra $\Lambda V$, we mean the largest $i$ for which $H^{i}(\Lambda V) \neq 0$. If $x$ is an element of a graded vector space, then we denote the degree of $x$ by $|x|$.

\section{Main Results}

We say that $\Lambda V$ has differential of homogeneous-length $l$ if $d: V \rightarrow \Lambda^{l} V$. A coformal minimal algebra has differential of homogeneous-length 2 . In the homogeneous-length differential case, the cohomology admits a second grading, $H^{+}(\Lambda V)=\oplus_{k \geq 1} H_{k}^{*}(\Lambda V)$, given by length of representative cocycle. Thus $x \neq$ $0 \in H_{k}^{*}(\Lambda V)$ if and only if $\mathrm{e}_{0}(x)=k$. We extend this second grading to include the degree zero component by setting $H_{0}^{*}(\Lambda V)$ equal to $\mathbb{Q}$ in degree zero, and zero elsewhere - see (1) below. Now introduce the following notation: If $H_{k}^{*}(\Lambda V) \neq 0$, then set

$$
n_{k}=\min \left\{i \mid H_{k}^{i}(\Lambda V) \neq 0\right\} \quad \text { and } \quad N_{k}=\max \left\{i \mid H_{k}^{i}(\Lambda V) \neq 0\right\} .
$$

We make some observations about the bigraded Poincaré duality algebra $H_{*}^{*}(\Lambda V)$. Denote $\mathrm{e}_{0}(\Lambda V)$ by $e$, so that $H^{*}(\Lambda V)=\oplus_{k=0}^{e} H_{k}^{*}(\Lambda V)$. By definition, we have

$$
H_{0}^{i}(\Lambda V)=\left\{\begin{array}{ll}
\mathbb{Q} & i=0 \\
0 & i>0
\end{array} \quad \text { so } \quad n_{0}=N_{0}=0 .\right.
$$

Now suppose that $H^{*}(\Lambda V)$ has formal dimension $N$. Since the second grading comes from length of representative cocycle, we have

$$
H_{e}^{i}(\Lambda V)=\left\{\begin{array}{ll}
\mathbb{Q} & i=N \\
0 & i<N
\end{array} \quad \text { so } \quad n_{e}=N_{e}=N .\right.
$$

We assemble our remaining remarks about $H_{*}^{*}(\Lambda V)$ into the following lemma. 
Lemma 2.1. Let $H$ be a bigraded Poincaré duality algebra of formal dimension $N$ that satisfies $H=\oplus_{k=0}^{e} H_{k}^{*}$ together with (1) and (2) above. Suppose that $H_{k}^{*} \neq 0$ for $k=1, \ldots, e-1$, and that $p$ is some positive integer. Then with the above notation, the following are equivalent:

(a) $n_{1}=p$ and $n_{k+1} \geq n_{k}+p$ for $k=1, \ldots, e-1$.

(b) $N_{k+1} \geq N_{k}+p$ for $k=0, \ldots, e-2$ and $N=N_{e}=N_{e-1}+p$.

Proof. Because $H$ is a bigraded algebra, and also satisfies Poincaré duality, it follows that there are non-degenerate pairings

$$
H_{k}^{i}(\Lambda V) \times H_{e-k}^{N-i}(\Lambda V) \rightarrow H_{e}^{N}(\Lambda V) \cong \mathbb{Q},
$$

for $k=1, \ldots, e-1$ and $i=1, \ldots, N-1$. Hence $n_{k}=N-N_{e-k}$ for $k=1, \ldots, e-1$. The equivalence of (a) and (b) follows.

To phrase the result, and to give its proof, we say that a graded vector space is $(i-1)$-connected if degree $i$ is the first non-zero degree. Also, for a bigraded cohomology algebra $H_{*}^{*}(\Lambda V)$ we shall refer to elements in $H_{*}^{i}(\Lambda V)$ as having upper degree $i$ and to elements in $H_{k}^{*}(\Lambda V)$ as having lower degree $k$. In our setting, upper degree corresponds to the usual topological degree, and lower degree corresponds to the Toomer invariant of a cohomology class. In this bigraded setting the notation $|x|$ still denotes the upper degree of the element $x$.

TheOREm 2.2. Suppose $\Lambda V$ is an elliptic minimal algebra with homogeneouslength l differential. Set

$$
e=\operatorname{dim} V^{\text {odd }}+(l-2) \operatorname{dim} V^{\text {even }} .
$$

Then we have

(A) $\operatorname{cat}_{0}(\Lambda V)=\mathrm{e}_{0}(\Lambda V)=e$;

(B) $H_{k}^{*}(\Lambda V) \neq 0$ for each $k=0, \ldots, e$;

(C) Suppose that $V$ is $(p-1)$-connected. Then the bigraded Poincaré duality algebra $H(\Lambda V)$ satisfies the two equivalent conditions of Lemma 2.1.

Proof. We will prove that $\mathrm{e}_{0}(\Lambda V)=e$. We can include cat ${ }_{0}(\Lambda V)$ in the statement of part (A) due to the result cat ${ }_{0}(\Lambda V)=\mathrm{e}_{0}(\Lambda V)$ for elliptic minimal algebras [FHL98].

Write $\Lambda V=\Lambda\left(x_{1}, \ldots, x_{n}\right)$, with $p=\left|x_{1}\right| \leq\left|x_{2}\right| \leq \cdots \leq\left|x_{n}\right|$ and $d\left(x_{1}\right)=0$. We argue by induction on the number of generators $n$. For $n=1$ ellipticity requires that $\left|x_{1}\right|$ be odd. In this case $e=1$ and all parts of the result are trivial.

Now assume inductively that the result holds for all elliptic minimal algebras with homogeneous-length differential and fewer than $n$ generators. Let $(\Lambda W ; \bar{d})=$ $\Lambda\left(x_{2}, \ldots, x_{n} ; \bar{d}\right)$ be the quotient obtained by factoring out the DG ideal generated by the generator $x_{1}$. Then $\Lambda W$ is elliptic [Hal77, Prop.1] and has $n-1$ generators. Note that $W$ also has differential of homogeneous-length $l$. Both $\Lambda V$ and $\Lambda W$ are bigraded DG algebras, with the lower grading in each given by word-length. Thus their cohomology algebras are bigraded in the sense discussed above. We will make extensive use of this bigraded structure without further remark.

Set $f=\operatorname{dim} W^{\text {odd }}+(l-2) \operatorname{dim} W^{\text {even }}$, so that we have

$$
e= \begin{cases}f+1 & \text { if }\left|x_{1}\right| \text { is odd } \\ f+(l-2) & \text { if }\left|x_{1}\right| \text { is even }\end{cases}
$$


Let $M$ and $N$ denote the formal dimensions of $\Lambda W$ and $\Lambda V$ respectively. Although we do not use it here, we mention that there is a formula that describes these formal dimensions in terms of the degrees of the generators [Hal77, Th.3].

Further, set $m_{k}=\min \left\{i \mid H_{k}^{i}(\Lambda W) \neq 0\right\}$ and $M_{k}=\max \left\{i \mid H_{k}^{i}(\Lambda W) \neq 0\right\}$, for $k=0, \ldots, f$. From the induction hypothesis, we have $\mathrm{e}_{0}(\Lambda W)=f, H_{k}^{*}(\Lambda W) \neq 0$ for $k=0, \ldots, f$ and $H_{*}^{M}(\Lambda W)=H_{f}^{*}(\Lambda W)=H_{f}^{M}(\Lambda W) \cong \mathbb{Q}$. Also, $m_{1}=\left|x_{2}\right|$ and $m_{k+1} \geq m_{k}+\left|x_{2}\right|$ for $k=1, \ldots, f-1$. Equivalently, as per Lemma 2.1, we have $M_{k+1} \geq M_{k}+\left|x_{2}\right|$ for $k=0, \ldots, f-2$ and $M=M_{f}=M_{f-1}+\left|x_{2}\right|$. To prove the induction step there are two cases, according as the parity of the degree of the first generator $x_{1}$.

Case I. $\left|x_{1}\right|=2 r+1$ is odd: In this case $e=f+1$. Here we form the following short exact sequence of DG vector spaces:

$$
0 \longrightarrow \Lambda W \stackrel{j}{\longrightarrow} \Lambda V \stackrel{p}{\longrightarrow} \Lambda W \longrightarrow 0 .
$$

Here, $p$ denotes the projection and $j$ denotes the degree- $(2 r+1)$ linear map defined by

$$
j(\chi)=(-1)^{|\chi|} x_{1} \chi
$$

for $\chi \in \Lambda W$. On cohomology, it is clear that $j^{*}$ increases lower degree by 1 , whilst $p^{*}$ preserves lower degree. The corresponding long exact sequence in cohomology has connecting homomorphism described as follows: Suppose $\chi \in \Lambda W$ is any element. Regard $\chi$ as an element of $\Lambda V$ and write $d \chi=\bar{d} \chi+x_{1} \theta(\chi)$. This defines a derivation $\theta: \Lambda W \rightarrow \Lambda W$, of degree- $(-2 r)$, that satisfies $\bar{d} \theta=\theta \bar{d}$. Hence we have an induced derivation on cohomology $\theta^{*}: H^{i}(\Lambda W) \rightarrow H^{i-2 r}(\Lambda W)$, also of degree$(-2 r)$. The derivation $\theta^{*}$ increases lower degree by $(l-2)$. The resulting long exact cohomology sequence (the Wang sequence) is therefore a long exact sequence of bigraded cohomology groups as follows:

$$
\text { (4) } \cdots H_{k-1-(l-2)}^{i-1}(\Lambda W) \stackrel{\theta^{*}}{\longrightarrow} H_{k-1}^{i-2 r-1}(\Lambda W) \stackrel{j^{*}}{\longrightarrow} H_{k}^{i}(\Lambda V) \stackrel{p^{*}}{\longrightarrow} H_{k}^{i}(\Lambda W) \cdots \text {. }
$$

It follows immediately from this long exact sequence that $H_{*}^{i}(\Lambda V)=0$ for $i \geq$ $M+2 r+2$ and $H_{k}^{*}(\Lambda V)=0$ for $k \geq f+2=e+1$. Furthermore, the long exact sequence restricts to isomorphisms

$$
0 \longrightarrow H_{k}^{M}(\Lambda W) \stackrel{j^{*}}{\cong} H_{k+1}^{M+2 r+1}(\Lambda V) \longrightarrow 0 .
$$

One sees from this that the formal dimensions of $\Lambda V$ and $\Lambda W$ are related by $N=M+2 r+1$ (cf. [FH82, Th.10.4]). This relation is also evident from the formula of Halperin referred to above. One also sees that $\mathrm{e}_{0}(\Lambda V)=f+1=e$. This proves part (A).

Now consider part (B). In the previous paragraph, we showed that $H_{*}^{N}(\Lambda V)=$ $H_{e}^{*}(\Lambda V)=H_{e}^{N}(\Lambda V) \cong \mathbb{Q}$. It is automatic that $H_{*}^{0}(\Lambda V)=H_{0}^{*}(\Lambda V)=H_{0}^{0}(\Lambda V) \cong$ $\mathbb{Q}$. We consider the remaining values $k=1, \ldots, e-1$. Denote the map

$$
\theta^{*}: H_{k}^{*}(\Lambda W) \rightarrow H_{k+(l-2)}^{*}(\Lambda W)
$$

by $\theta_{k}^{*}$. From the bigraded Wang sequence (4) we obtain a short exact sequence

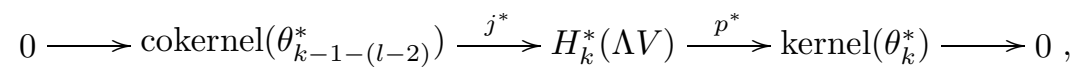


for each $k=1, \ldots, f$. From the inductive hypothesis applied to $\Lambda W$, we have

$$
m_{k+(l-2)} \geq m_{k}+(l-2)\left|x_{2}\right| \geq m_{k}
$$

and

$$
M_{k+(l-2)} \geq M_{k}+(l-2)\left|x_{2}\right| \geq M_{k} .
$$

Since $\theta_{k}^{*}$ is of negative degree, (7) and (8) imply respectively that both $\operatorname{kernel}\left(\theta_{k}^{*}\right)$ and cokernel $\left(\theta_{k-1-(l-2)}^{*}\right)$ are non-zero for $k=1, \ldots, f$. It follows from $(6)$ that $\operatorname{dim}\left(H_{k}^{*}(\Lambda V)\right) \geq 2$ for each $k=1, \ldots, f=e-1$.

Finally, we establish $(\mathrm{C})$ in the present case, by showing that $H_{*}^{*}(\Lambda V)$ satisfies condition (a) of Lemma 2.1 with $p=\left|x_{1}\right|$. We observe from the sequence (6) that $H_{k}^{i}(\Lambda V)=0$ for $i<m_{k-1}+\left|x_{1}\right|$. For if $i<m_{k-1}+\left|x_{1}\right| \leq m_{k-1}+\left|x_{2}\right|$, it follows that there can be no contribution to $H_{k}^{i}(\Lambda V)$ from cokernel $\left(\theta_{k-1-(l-2)}^{*}\right)$. On the other hand, $i<m_{k-1}+\left|x_{1}\right|$ implies $i<m_{k}$. Thus if $i<m_{k-1}+\left|x_{1}\right|$, then neither does kernel $\left(\theta_{k}^{*}\right)$ contribute to $H_{k}^{i}(\Lambda V)$. So we have the inequality

$$
n_{k} \geq m_{k-1}+\left|x_{1}\right|
$$

for $k=l, \ldots, f=e-1$. Next, recall that $\theta_{k}^{*}$ is of negative degree. Hence $\operatorname{kernel}\left(\theta_{k}^{*}\right)$ begins in degree $m_{k}$. From (6), therefore, we obtain

$$
n_{k} \leq m_{k}
$$

for $k=l, \ldots, f=e-1$. Combining the inequalities (9) and (10), with a shift of subscript in the latter, gives

$$
n_{k} \geq m_{k-1}+\left|x_{1}\right| \geq n_{k-1}+\left|x_{1}\right|
$$

for $k=2, \ldots, f=e-1$. In addition, it is evident that $n_{1}=\left|x_{1}\right|$. From (5), we see that $n_{f+1}=m_{f}+\left|x_{1}\right| \geq n_{f}+\left|x_{1}\right|$, with the latter inequality obtained from (10). This establishes (C) and completes the induction step in Case I.

Case II. $\left|x_{1}\right|=2 r$ is even: In this case $e=f+(l-2)$. Here, consider the short exact sequence (again of DG vector spaces) as follows:

$$
0 \longrightarrow \Lambda V \stackrel{j}{\longrightarrow} \Lambda V \stackrel{p}{\longrightarrow} \Lambda W \longrightarrow 0,
$$

where $p$ denotes the projection and $j$ the degree- $(2 r)$ map defined by $j(\chi)=x_{1} \chi$ for $\chi \in \Lambda V$. The corresponding long exact sequence in cohomology has connecting homomorphism as follows: Suppose $\bar{d} \chi=0$ for $\chi \in \Lambda W$, so that $d \chi=x_{1} \chi^{\prime}=j\left(\chi^{\prime}\right)$ for some $\chi^{\prime} \in \Lambda V$ and then $\partial^{*}([\chi])=\left[\chi^{\prime}\right]$. Here $\partial^{*}$ is of degree- $(-2 r+1)$ and if $\chi \in \Lambda^{\geq k} W$, then $\chi^{\prime} \in \Lambda^{\geq k+(l-2)} V$. On passing to (bigraded) cohomology, $\partial^{*}$ increases lower degree by $(l-2), p^{*}$ preserves lower degree and $j^{*}$ increases lower degree by one. In this case, therefore, the resulting long exact sequence of bigraded cohomology groups (the Gysin sequence) is as follows:

$$
\cdots H_{k-1}^{i-2 r}(\Lambda V) \stackrel{j^{*}}{\longrightarrow} H_{k}^{i}(\Lambda V) \stackrel{p^{*}}{\longrightarrow} H_{k}^{i}(\Lambda W) \stackrel{\partial^{*}}{\longrightarrow} H_{k+(l-2)}^{i-2 r+1}(\Lambda V) \cdots .
$$

For $i \geq M+2$, this sequence gives isomorphisms

$$
0 \longrightarrow H_{*}^{i-2 r}(\Lambda V) \stackrel{j^{*}}{\cong} H_{*}^{i}(\Lambda V) \longrightarrow 0 .
$$

Since $H_{*}^{i}(\Lambda V)$ must be zero for sufficiently large $i$, it follows that $N \leq M-2 r+1$ and that $\partial^{*}: H_{*}^{M}(\Lambda W) \rightarrow H_{*}^{M-2 r+1}(\Lambda V)$ is an isomorphism. Thus we obtain $N=M-2 r+1$ (cf. [FH82, Th.10.4]). Once again, this relation is also evident from 
the formula of Halperin referred to above. On the other hand, for $k \geq f+(l-2)+2$, (11) gives isomorphisms

$$
0 \longrightarrow H_{k-1}^{*}(\Lambda V) \stackrel{j^{*}}{\cong} H_{k}^{*}(\Lambda V) \longrightarrow 0
$$

Again, since $H_{k}^{*}(\Lambda V)$ must also be zero for sufficiently large $k$, it follows that $\mathrm{e}_{0}(\Lambda V) \leq f+(l-2)=e$. The isomorphism $\partial^{*}$ restricts to isomorphisms

$$
0 \stackrel{p^{*}}{\longrightarrow} H_{k}^{M}(\Lambda W) \stackrel{\partial^{*}}{\cong} H_{k+(l-2)}^{M-2 r+1}(\Lambda V) \stackrel{j^{*}}{\longrightarrow} 0 .
$$

It follows that $\mathrm{e}_{0}(\Lambda V)=f+(l-2)=e$, as desired for part (A).

For parts $(\mathrm{B})$ and $(\mathrm{C})$, we show $H_{k}^{*}(\Lambda V) \neq 0$ for $k=1, \ldots, e$ and establish condition (b) of Lemma 2.1, by using a (secondary) induction on $k$.

The secondary induction hypothesis is as follows:

(i) $H_{i}^{*}(\Lambda V) \neq 0$ for $i=1, \ldots, k-1$,

(ii) $N_{i} \geq N_{i-1}+2 r$ for $i=1, \ldots, k-1$, and

(iii) $N_{i} \geq M_{i-(l-2)}-2 r+1$, for $i=l-1, \ldots, k-1$.

This induction starts with $k=l$. Since the differential is of length $l$, there are no boundaries of length $\leq(l-1)$. Therefore $x_{1}^{i} \neq 0 \in H_{i}^{2 r i}(\Lambda V)$ for $i=1, \ldots, l-1$. Furthermore, if $\alpha \neq 0 \in H_{i}^{N_{i}}(\Lambda V)$ for $i=1, \ldots, l-2$, then $x_{1} \alpha \neq 0 \in H_{i+1}^{N_{i}+2 r}(\Lambda V)$, again because there are no boundaries of length $\leq(l-1)$. Hence $N_{i} \geq N_{i-1}+2 r$ for $i=1, \ldots, l-1$. This establishes (i) and (ii) with $k=l$. For (iii), we must show that $N_{l-1} \geq M_{1}-2 r+1$. So consider the following portion of the Gysin sequence:

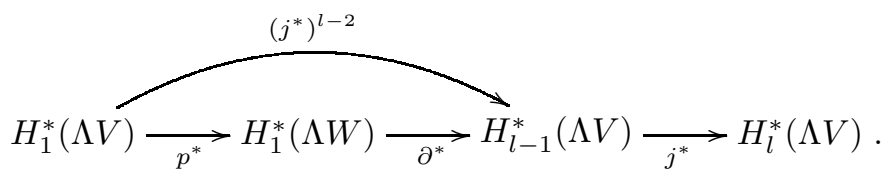

The map $\left(j^{*}\right)^{l-2}: H_{1}^{*}(\Lambda V) \rightarrow H_{l-1}^{*}(\Lambda V)$ is simply $j^{*}$ composed with itself $(l-2)$ times. In other words, this map is multiplication by the class of $x_{1}(l-2)$-times. Note that the diagram as displayed is not commutative. By our primary induction hypothesis, we have some $\alpha \neq 0 \in H_{1}^{M_{1}}(\Lambda W)$. If $\partial^{*}(\alpha) \neq 0 \in H_{l-1}^{M_{1}-2 r+1}(\Lambda V)$, then $N_{l-1} \geq M_{1}-2 r+1$, and (iii) holds for $k=l$. On the other hand, if $\partial^{*}(\alpha)=0$, then exactness provides some $\beta \neq 0 \in H_{1}^{M_{1}}(\Lambda V)$ with $p^{*}(\beta)=\alpha$. Then $\left(j^{*}\right)^{l-2}(\beta) \neq$ $0 \in H_{l-1}^{M_{1}+2 r(l-2)}(\Lambda V)$, once more because there are no boundaries of length $\leq l-1$. Since $\left|\left(j^{*}\right)^{l-2}(\beta)\right|=M_{1}+2 r(l-2)>M_{1}-2 r+1$, we have shown (iii) for $k=l$ under either of the two possible circumstances. This starts our induction.

Now we make the inductive step. Given the secondary inductive hypothesis for some $k \geq l$, we will show (i), (ii) and (iii) for $i=k$. Observe, that in any one Gysin sequence, some terms cannot appear due to the non-consecutive nature of the indices. We take the following two versions of the Gysin sequence, each of 
which features $H_{k}^{*}(\Lambda V)$ :

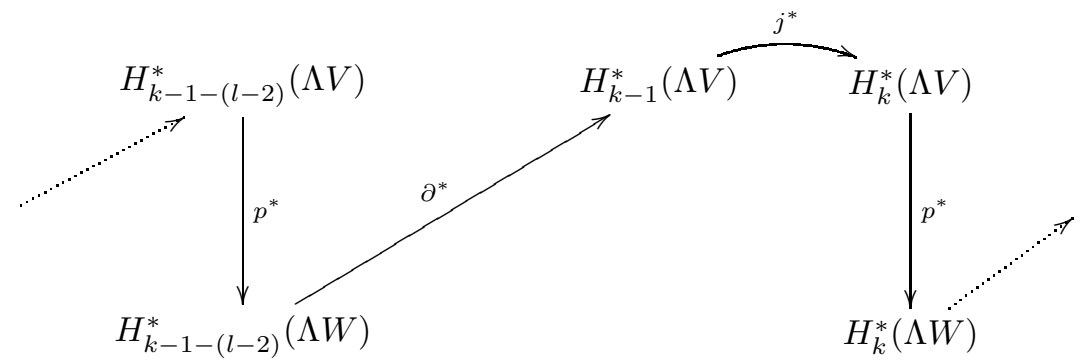

and

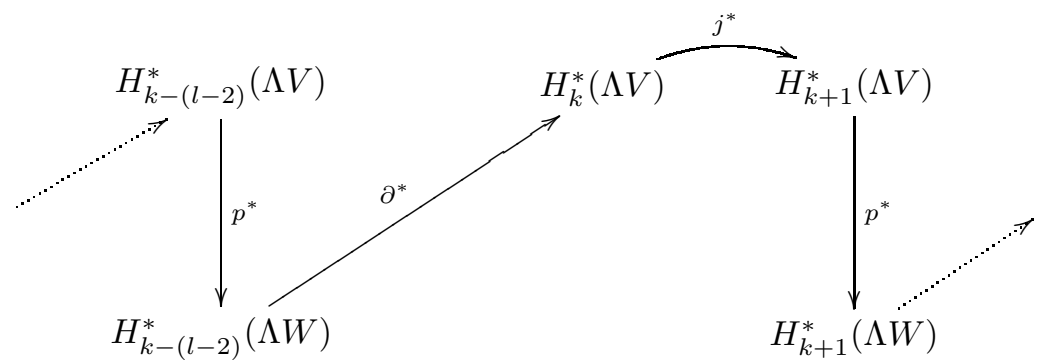

We splice these together at the $H_{k}^{*}(\Lambda V)$ term, then add the map $\left(j^{*}\right)^{l-2}=j^{*}$ 。 $\left(j^{*}\right)^{l-3}: H_{k-(l-2)}^{*}(\Lambda V) \rightarrow H_{k}^{*}(\Lambda V)$, as we did in (12) when starting this induction. This gives the following diagram, that contains the maps to which we refer in our argument.

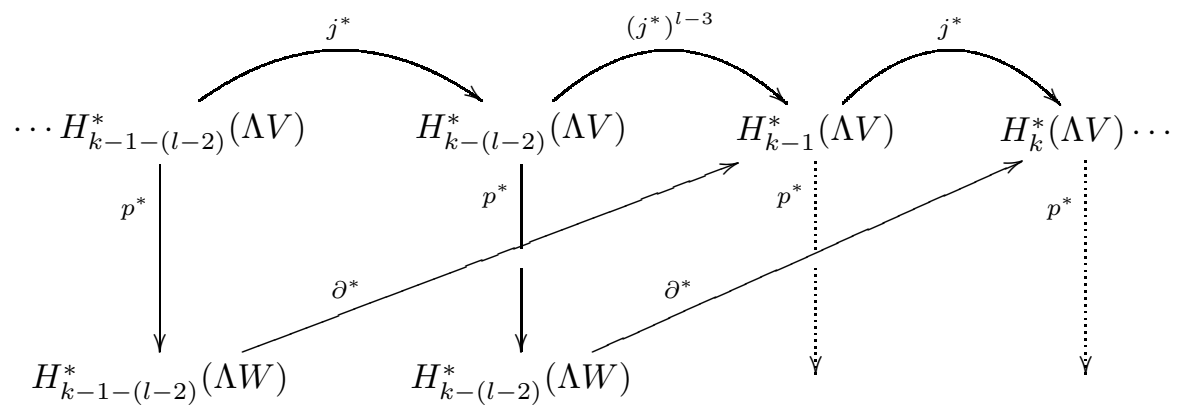

We note again that this is not a commutative diagram.

Our primary inductive hypothesis gives some $\alpha \neq 0 \in H_{k-(l-2)}^{M_{k-(l-2)}}(\Lambda W)$. If $\partial^{*}(\alpha) \neq 0 \in H_{k}^{M_{k-(l-2)}-2 r+1}(\Lambda V)$, then $N_{k} \geq M_{k-(l-2)}-2 r+1$, and (iii) holds for $i=k$. On the other hand, if $\partial^{*}(\alpha)=0$, then exactness provides some $\beta \neq 0 \in H_{k-(l-2)}^{M_{k-(l-2)}}(\Lambda V)$ with $p^{*}(\beta)=\alpha$. We claim that $\left(j^{*}\right)^{l-2}(\beta) \neq 0 \in$ $H_{k}^{M_{k-(l-2)}+2 r(l-2)}(\Lambda V)$. This claim follows from a combination of exactness and degree considerations. For the case $l=2$, we interpret $\left(j^{*}\right)^{0}(\beta)$ as $\beta$. For $l \geq 3$, Consider the sequence of elements $\left\{\beta, j^{*}(\beta), \ldots,\left(j^{*}\right)^{t}(\beta), \ldots,\left(j^{*}\right)^{l-2}(\beta)\right\}$. By exactness, we have

$$
\begin{aligned}
\operatorname{ker}\left(\left(j^{*}\right):\right. & \left.H_{k-(l-2)+(t-1)}^{*}(\Lambda V) \rightarrow H_{k-(l-2)+t}^{*}(\Lambda V)\right) \\
& =\operatorname{im}\left(\left(\partial^{*}\right): H_{k-2(l-2)+(t-1)}^{*}(\Lambda W) \rightarrow H_{k-(l-2)+(t-1)}^{*}(\Lambda V)\right)
\end{aligned}
$$


for $t=1, \ldots, l-2$. Now $\partial^{*}\left(H_{k-2(l-2)+(t-1)}^{*}(\Lambda W)\right)$ is zero in upper degrees above degree $M_{k-2(l-2)+(t-1)}-2 r+1$. Since $\left|\left(j^{*}\right)^{t-1}(\beta)\right|=M_{k-(l-2)}+2 r(t-1)$, and since our primary induction hypothesis gives

$$
M_{k-(l-2)} \geq M_{k-2(l-2)+(t-1)}+((l-2)-(t-1))\left|x_{2}\right|,
$$

it follows that $\left(j^{*}\right)^{t-1}(\beta)$ is not in $\operatorname{ker}\left(j^{*}\right)$ for $t=1, \ldots, l-2$. From the claim, we have $N_{k} \geq M_{k-(l-2)}+2 r(l-2)>M_{k-(l-2)}-2 r+1$. Under either of the two possible circumstances, therefore, we have shown (i) and (iii) for $i=k$.

We complete the inductive step by showing (ii) for $i=k$. Our secondary induction hypothesis provides an element $\gamma \neq 0 \in H_{k-1}^{N_{k-1}}(\Lambda V)$. If $j^{*}(\gamma) \neq 0 \in$ $H_{k}^{N_{k-1}+2 r}(\Lambda V)$, then $N_{k} \geq N_{k-1}+2 r$, and (ii) holds for $i=k$. On the other hand, if $j^{*}(\gamma)=0$, then exactness implies $\gamma=\partial^{*}(\delta)$, for some $\delta \neq 0 \in H_{k-1-(l-2)}^{N_{k-1}+2 r-1}(\Lambda W)$. This implies $M_{k-1-(l-2)} \geq N_{k-1}+2 r-1$ which, combined with (iii) of the secondary induction hypothesis gives $M_{k-1-(l-2)}=N_{k-1}+2 r-1$. Combining this equality with (iii) for $i=k$, which we just established, and the primary induction hypothesis, we have

$$
\begin{aligned}
N_{k} & \geq M_{k-(l-2)}-2 r+1 \\
& \geq M_{k-(l-2)}+\left|x_{2}\right|-2 r+1 \\
& =N_{k-1}+\left|x_{2}\right| \geq N_{k-1}+\left|x_{1}\right| .
\end{aligned}
$$

This establishes (ii) in the case $i=k$ and hence completes our secondary induction step.

To complete the primary induction step in Case II, it only remains to show $N_{e}=N_{e-1}+\left|x_{1}\right|$. To see this, observe that $H_{*}^{*}(\Lambda V)$ is a bigraded Poincaré duality algebra that satisfies, in particular, $n_{1}=N-N_{e-1}$. Clearly $n_{1}=\left|x_{1}\right|$ and so $N=N_{e-1}+\left|x_{1}\right|$. This completes the (primary) induction step in Case II.

In both Case I and Case II the induction has been completed and this proves the result.

REMARK 2.3. In the above, we referred to (4) as a Wang sequence and (11) as a Gysin sequence. In Case I of the proof, we have a fibration sequence of minimal models $\Lambda\left(x_{1}\right) \longrightarrow \Lambda V \longrightarrow \Lambda W$. Topologically, this corresponds to a fibration sequence of spaces $F \rightarrow E \rightarrow S^{2 r+1}$, with base an odd-dimensional sphere. The Wang sequence of this fibration, in the usual sense [Whi78, p.319], corresponds to (4). In Case II, our fibration sequence of minimal models corresponds topologically to a fibration sequence of spaces $F \rightarrow E \rightarrow K(\mathbb{Q}, 2 r)$. Going one step back in the fibre sequence gives a fibration sequence $K(\mathbb{Q}, 2 r-1) \rightarrow F \rightarrow E$. Since $K(\mathbb{Q}, 2 r-1)$ and $S^{2 r-1}$ have the same rational homotopy type, we end up rationally with a fibration sequence $S^{2 r-1} \rightarrow F \rightarrow E$, with fibre an odd-dimensional sphere. The Gysin sequence of this fibration [Whi78, p.357], corresponds to (11).

REMARK 2.4. In the introduction, we mentioned that the formula in part (A) of Theorem 2.2 can be adapted into a lower bound for the rational category of any elliptic space. Specifically, this goes as follows: We say $\Lambda V$ has differential of length at least $l$ if $d: V \rightarrow \Lambda^{\geq l} V$. Then with $e$ as in the statement of Theorem 2.2, the inequality cat ${ }_{0}(\Lambda V)=\mathrm{e}_{0}(\Lambda V) \geq e$ holds for any elliptic space. Once again, this is Corollary 3 of [GJ01]. The proof of Theorem 2.2 is easily adapted to establish this inequality. One simply omits all reference to parts (B) and (C) of the proof, and uses the Wang and Gysin sequences in their ordinary, i.e., not bigraded, versions 
in precisely the same way as they were used above. Likewise, the proof of part (A) of Theorem 2.2 can be given independently of the proof of parts $(\mathrm{B})$ and $(\mathrm{C})$. It is interesting to note, however, that the proofs of these latter two parts cannot be separated from each other.

For our last result, we add the hypothesis that $\operatorname{ker}\left(d: V^{\text {odd }} \rightarrow \Lambda V\right)$ is nonzero to those of Theorem 2.2. This extra hypothesis admits a simple topological translation. Namely, that the rational Hurewicz homomorphism $h: \pi_{*}(X) \otimes \mathbb{Q} \rightarrow$ $H_{*}(X ; \mathbb{Q})$ is non-zero in some odd degree.

TheOREM 2.5. Suppose $\Lambda V$ is an elliptic minimal algebra with homogeneouslength $l$ differential and $\operatorname{ker}\left(d: V^{\text {odd }} \rightarrow \Lambda V\right)$ non-zero. Then $\operatorname{dim}\left(H_{k}^{*}(\Lambda V)\right) \geq 2$ for each $k=1, \ldots, e-1$, where $e=\operatorname{cat}_{0}(\Lambda V)=\mathrm{e}_{0}(\Lambda V)$ is given by the formula of Theorem 2.2.

Proof. Suppose $u \in V^{\text {odd }}$ is a cocycle. Then we can write $\Lambda V=\Lambda\left(x_{1}, \ldots, x_{n}\right)$ with $x_{1}=u$. Now argue exactly as in case I of the proof of Theorem 2.2. Observe that our quotient $\Lambda W=\Lambda\left(x_{2}, \ldots, x_{n} ; \bar{d}\right)$ in the present result satisfies the hypotheses of Theorem 2.2. Therefore, the part of the argument featuring the short exact sequence (6), with the inequalities (9) and (10), used there to establish the inductive step, can be used here to conclude our result.

COROLlary 2.6. Let $X$ be an elliptic space whose minimal model has a homogeneous-length differential and whose rational Hurewicz homomorphism is non-zero in some odd degree. Then $\operatorname{dim}\left(H^{*}(X ; \mathbb{Q})\right) \geq 2 \operatorname{cat}_{0}(X)=2 \mathrm{e}_{0}(X)$.

Proof. Let $\Lambda V$ denote the minimal model of $X$. From Theorem 2.5, we obtain $\operatorname{dim}\left(H^{*}(\Lambda V)\right) \geq 2 \operatorname{cat}_{0}(\Lambda V)=2 \mathrm{e}_{0}(\Lambda V)$. The statements about $X$ follow from the standard translation from minimal models to spaces.

\section{Examples and Comments}

There are a number of special cases of our main results, which either retrieve well-known results or provide interesting examples.

Example 3.1. Consider the case in which $l=2$. Part (A) of Theorem 2.2 specializes to retrieve part of [FH82, Prop.10.6], where it is shown that $\mathrm{e}_{0}(\Lambda V)=$ $\operatorname{dim} V^{\text {odd }}$ for $\Lambda V$ elliptic and coformal.

ExAmPle 3.2. Consider the case in which $\Lambda V=\Lambda\left(x_{1}, \ldots, x_{n}\right)$ is a minimal algebra with $\left|x_{i}\right|=1$ for each $i$. This type of example arises as the minimal model of a nilmanifold [Opr92]. Although we are primarily interested in the simply connected case, the results proved here carry though verbatim for this nilmanifold case. Here $\mathrm{e}_{0}(x)=i$ if and only if $x \neq 0 \in H^{i}(\Lambda V)$. Thus the Toomer invariant of a cohomology class is identified with the degree and an integer $i$ is realized as the Toomer invariant of some class if and only $b_{i} \neq 0$, where $b_{i}$ denotes the $i$ 'th Betti number of $\Lambda V$, or of the nilmanifold of which $\Lambda V$ is the minimal model.

For degree reasons, the differential here must be homogeneous of length 2 . Therefore Theorem 2.5 and Corollary 2.6 specialize to yield the following wellknown result, which is essentially due to Dixmier [Dix55]:

Proposition 3.3. A nilmanifold $X$ of dimension $n$ has $b_{i} \geq 2$ for $1 \leq i \leq n-1$ and hence $\operatorname{dim}\left(H^{*}(X ; \mathbb{Q})\right) \geq 2 \operatorname{dim}(X)$. 
The conclusion of Theorem 2.5 obviously holds in many cases besides those covered by the hypotheses. We cannot resist making the following conjecture:

CONJeCture 3.4. Let $\Lambda V$ be elliptic with homogeneous-length differential. Either $\operatorname{dim}\left(H_{k}^{*}(\Lambda V)\right) \geq 2$ for each $k=1, \ldots, e-1$, where $e=\operatorname{cat}_{0}(\Lambda V)=\mathrm{e}_{0}(\Lambda V)$ is given by the formula of Theorem 2.2, or $H^{*}(\Lambda V)$ is a truncated polynomial algebra on a single generator.

In view of Theorem 2.5 , one need only consider the case in which $\operatorname{ker}(d: V \rightarrow$ $\Lambda V$ ) is concentrated in even degrees. We believe that an argument as in case II of the proof of Theorem 2.2, together with a careful analysis of the exceptional cases, will establish Conjecture 3.4 at least in the coformal case, $l=2$. We have been unable to prove the general case, however, using this approach.

It is also clear that in many cases to which Theorem 2.5 applies, and others to which it does not, the inequalities $\operatorname{dim}\left(H_{k}^{*}(\Lambda V)\right) \geq 2$ for each $k=1, \ldots, e-1$ and $\operatorname{dim}\left(H^{*}(X ; \mathbb{Q})\right) \geq 2 \operatorname{cat}_{0}(X)$ are by no means sharp. The following examples show that, nonetheless, these inequalities are best possible for a general result of this nature.

ExAmple 3.5. Let $X_{l}=\mathbb{C} P^{l-1} \times S^{2 r+1}$ for $l \geq 2$. Then $X_{l}$ is elliptic and has minimal model with homogeneous-length $l$ differential. As is well known, $\operatorname{cat}_{0}\left(X_{l}\right)=\mathrm{e}_{0}\left(X_{l}\right)=l+1$. On the other hand, we see that $\operatorname{dim}\left(H_{k}^{*}(X ; \mathbb{Q})\right)=2$ for $k=1, \ldots, l$. Thus we have $\operatorname{dim}\left(H^{*}\left(X_{l} ; \mathbb{Q}\right)\right)=2 \operatorname{cat}_{0}\left(X_{l}\right)$.

Example 3.6. Let $\Lambda V=\Lambda\left(x_{1}, x_{2}, y_{1}, y_{2}, y_{3}\right)$ with $\left|x_{1}\right|=\left|x_{2}\right|=2,\left|y_{1}\right|=$ $\left|y_{2}\right|=\left|y_{3}\right|=3$ and differential $d\left(x_{1}\right)=0=d\left(x_{2}\right), d\left(y_{1}\right)=x_{1}^{2}, d\left(y_{2}\right)=x_{1} x_{2}$ and $d\left(y_{3}\right)=x_{2}^{2}$. Then $\Lambda V$ is elliptic and coformal. As is easily checked, we have $\operatorname{cat}_{0}(\Lambda V)=\mathrm{e}_{0}(\Lambda V)=3$, and $\operatorname{dim}\left(H_{k}^{*}(\Lambda V)\right)=2$ for $k=1$, 2 . In particular, we have $\operatorname{dim}\left(H^{*}(\Lambda V)\right)=2 \operatorname{cat}_{0}(\Lambda V)$.

It would be interesting to know whether there are other examples in which the inequalities of Corollary 2.6 and Theorem 2.5 are sharp, or whether these examples are essentially the only such. In particular, it would be interesting to find general conditions under which the inequality of Corollary 2.6 could be (substantially) strengthened.

Finally, we remark that the original motivation for this work came from a question of Yves Félix, as to whether there exists any space with "e $e_{0}$-gaps" in its cohomology. Precisely, we say that a space $X$ has an "e $0_{0}$ gap" in its cohomology if $H^{*}(X, \mathbb{Q})$ has an element whose Toomer invariant is $k$, but does not have any element whose Toomer invariant is $k-1$. A recent example due to Kahl and Vandembroucq [KV01] shows that $\mathrm{e}_{0}$-gaps can occur in the cohomology of a finite complex. Their example is actually a Poincaré duality space, but it is hyperbolic and not elliptic. This leaves the following question:

QUESTION 3.7. Can an elliptic space have $\mathrm{e}_{0}$-gaps in its cohomology?

If it is not possible for an elliptic space to have $\mathrm{e}_{0}$-gaps in its cohomology, then it would seem reasonable to extend Conjecture 3.4 to the general elliptic space.

\section{References}

[CJ01] M. Cuvilliez and B. Jessup, The rational LS-category of $k$-trivial fibrations, To appear, Proc. Amer. Math. Soc., 2001. 
[Dix55] J. Dixmier, Cohomologie des algèbres de Lie nilpotentes, Acta Sci. Math. Szeged 16 (1955), 246-250.

[FH82] Y. Félix and S. Halperin, Rational LS-category and its applications, Trans. Amer. Math. Soc. 273 (1982), 1-37.

[FHL98] Y. Félix, S. Halperin, and J.-M. Lemaire, The rational LS category of products and of Poincaré duality complexes, Topology 37 (1998), 749-756.

[FHT01] Y. Félix, S. Halperin, and J.-C. Thomas, Rational homotopy theory, Graduate Texts in Mathematics, vol. 205, Springer-Verlag, New York, 2001.

[GJ01] S. Ghorbal and B. Jessup, Estimating the rational LS-category of elliptic spaces, Proc. Amer. Math. Soc. 129 (2001), 1833-1842.

[Hal77] S. Halperin, Finiteness in the minimal models of Sullivan, Trans. Amer. Math. Soc. 230 (1977), 173-199.

[KV01] T. Kahl and L. Vandembroucq, Gaps in the Milnor-Moore spectral sequence, pre-print, 2001.

[LM01] L. Lechuga and A. Murillo, A formula for the rational LS-category of certain spaces, To appear, Annales de l'Inst. Fourier.

[Opr92] J. Oprea, The category of nilmanifolds, Enseign. Math. 38 (1992), 27-40.

[Rud99] Yu. B. Rudyak, On category weight and its applications. Topology 38 (1999) no. 1, $37-55$.

[Str97] J. Strom, Category weight and essential category weight, Thesis, Univ. of Wisconsin 1997.

[Whi78] G.W. Whitehead, Elements of homotopy theory, Graduate Texts in Mathematics, vol. 61, Springer-Verlag, New York, 1978.

Department of Mathematics, Cleveland State University, Cleveland OH 44115

E-mail address: Lupton@math.csuohio.edu 